\section{Hat die Radikaloperation als Methode versagt?}

\author{
Früherkennung kann auch schaden - etwa wenn ein frisch diagnostiziertes \\ Prostatakarzinom niedriger Malignität operiert wird, obwohl der Krebs für \\ den Patienten nie negativ in Erscheinung getreten wäre. Die Wirksamkeit \\ von Operation und Beobachtung wurde in einer randomisierten Studie \\ verglichen.
}

D as geflügelte Wort vom „Haustierkrebs" hat längst die Runde gemacht: So manches Prostatakarzinom würde seinen Träger heimlich, still und ohne auffallend negative Folgen bis in den Tod begleiten, wenn es nicht zwischendurch wegen eines allzu hohen PSA-Spiegels auffallen würde. Mit Maßnahmen wie der Prostatektomie würde bei diesen niedrig-malignen, vergleichsweise harmlosen Neoplasien daher quasi wie mit Kanonen auf Spatzen geschossen, meinen Kritiker: Der Kollateralschaden sei gigantisch, der Nutzen minimal. Sie plädieren daher seit Jahren dafür, gerade ein Prostatakarzinom im Frühstadium, das nur durch einen hohen PSA-Spiegel aufgefallen ist, zunächst nicht zu operieren, sondern einfach zu beobachten.

Die lang erwarteten Ergebnisse einer randomisierten Studie zeigen nun, welche Konsequenzen die Beobachtung im Vergleich zur Prostatektomie für das Wohlergehen der Patienten hat: Zwischen November 1994 und Januar 2002 waren für die Studie 731 Männer mit lokalisiertem Prostatakarzinom randomisiert worden. Ihr medianer PSA-Wert lag bei 7,8 ng/ml. Eine Gruppe unterzog sich daraufhin einer radikalen Prostatektomie, die andere wurde nur beobachtet. Primärer Endpunkt war die Mortalität in den Gruppen.

Während des medianen Follow-ups von 10,0 Jahren starben in der Prostatektomie-Gruppe 171 der 364 Männer (47,0\%); in der Beobachtungsgruppe 183 von 367 (49,9\%). So ergab sich eine Hazard Ratio [HR] von 0,88 .

Unter den Probanden, die der radikalen Prostatektomie (RPE) zugelost waren, starben 21 (5,8\%) infolge eines Prostatakrebses oder der Behandlung; dies war bei 31 Männern (8,4\%) in der Beobachtungsgruppe der Fall (HR 0,63; $\mathrm{p}=$ 0,09). Der Einfluss der Behandlung auf die Gesamtmortalität und die Prostata-
karzinom-Mortalität unterschied sich auch dann nicht signifikant, wenn Alter, ethnische Zugehörigkeit, Komorbiditäten, der selbstberichtete PerformanceStatus oder histologische Eigenschaften des Tumors einbezogen wurden.

Die radikale Prostatektomie war bei einem PSA-Spiegel von mehr als $10 \mathrm{ng} / \mathrm{ml}$ aber doch mit einer gesenkten Gesamtmortalität assoziiert. Dies mag auch für Tumoren mit mittlerem oder höherem Risiko gelten. Zu Nebenwirkungen kam es binnen 30 Tagen nach der Operation bei $21,4 \%$ der Männer, darunter war auch ein Todesfall.

Fazit: Unter Männern mit lokalisiertem Prostatakarzinom, deren Krebsdiagnose zu einem frühen Zeitpunkt ihrer Krankheit durch einen PSA-Test gestellt wurde, senkte eine radikale Prostatektomie nicht signifikant das Mortalitätsrisiko und auch nicht das Prostatakrebs-spezifische Mortalitätsrisiko im Vergleich zur alleinigen Beobachtung. Dies gilt zumindest für ein Follow-up von etwa zwölf Jahren.

Christina Berndt

Wilt TJ et al. Radical prostatectomy versus observation for localized prostate cancer. $\mathbf{N}$ Engl J Med. 2012;367(3):203-13.

Kommentar von Dr. Zimmermann: Ist der Prostatakrebs doch harmlos oder ist nur die operative Behandlung ineffektiv? Dieser provokanten Frage kann man angesichts der in der PIVOT-Studie vorgestellten sehr sorgfältig erhobenen Ergebnisse mittlerweile nicht mehr aus dem Wege gehen. 731 Männer mit vermutlich lokal begrenztem Prostatakarzinom wurden in einer sehr präzise angelegten Studie randomisiert und über eine mediane Follow-up-Zeit von 10,1 Jahren nachbeobachtet. Jeweils ca. $50 \%$ der beobachteten Männer mit einem mittleren Alter von 67 Jahren waren in diesem Zeitraum gestorben, 5,8 versus 8,4\% am Prostatakarzinom.
Zum Vergleich: laut den Sterbetafeln des statistischen Bundesamtes (die in den USA differieren nur wenig) hat ein 67-jähriger Mann eine durchschnittliche Lebenserwartung von 15,65 Jahren. Seine Wahrscheinlichkeit, zehn Jahre zu überleben, beträgt rund $73,4 \%$. Bei aller angebrachten Vorsicht lässt der Vergleich vom Outcome der PIVOTStudie mit den Sterbetafeln die Schlussfolgerung zu, dass die durchschnittliche Lebenserwartung sowohl in der Gruppe der beobachteten wie auch der behandelten Männer deutlich schlechter ist als von den Sterbetafeln prognostiziert.

Das bedeutet, dass die eingangs gestellte Frage eher nicht mit der Antwort "der Prostatakrebs ist harmlos" beantwortet werden darf. Die Betrachtung der Detailergebnisse verstärkt diese Vermutung: während bei den Niedrigrisiko-Patienten Gesamtvorteile im Überleben zugunsten der Active-Surveillance-Gruppe zu sehen waren, überwog der Vorteil in der Intermediate- und Hochrisiko-Gruppe doch - obgleich immer noch nicht das Signifikanzniveau erreicht wurde - zugunsten der operierten Patienten. Offensichtlich gibt es also behandlungsbedingte Übermortalitäten in der RPE-Gruppe und bei höherem Risiko ist nur eine geringe Effektivität zu beobachten.

Wahrscheinlich muss die Antwort auf die eingangs gestellte Frage vielmehr lauten, dass die alleinige operative Behandlung nicht nur zu leichten Übermortalitäten im Niedrigrisiko-Stadium führt, sondern insbesondere in allen Stadien nicht ausreichend effektiv ist. Dies ist auch mit den Ergebnissen der im Februar 2012 publizierten MetaAnalyse von Peter Grimm und Kollegen [Grimm P et al. BJU Int. 2012;109(Suppl 1): 22-9] in Einklang zu bekommen, hier fallen die Kurationsraten bei höherem Progressionsrisiko auf sehr niedrige Prozentsätze ab. Auch die hohen Sterberaten in den generellen Sterbestatistiken sprechen hierfür.

Über die detaillierten Ursachen der mangelhaften Effektivität der Radikaloperation im Vergleich zur Active-Surveillance wird in den nächsten Jahren zu diskutieren sein.

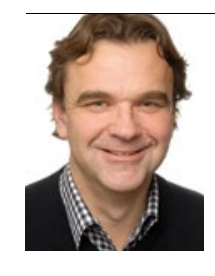
Dr. med. Jörg Zimmermann Arzt für Strahlentherapie Schwerpunkt Brachytherapie info@brachytherapiehamburg.de 